\title{
8. Gauss-Manin System and Mixed Hodge Structure
}

\author{
By Morihiko SAITo \\ Research Institute for Mathematical Sciences, Kyoto University \\ (Communicated by Kunihiko KodAIRA, M. J. A., Jan. 12, 1982)
}

Let $f: C^{n+1}, 0 \rightarrow C, 0$ be a holomorphic function with an isolated singularity. There are two ways of describing the degeneracy of a one-parameter family $\left\{X_{t}=f^{-1}(t)\right\}$. One is the theory of Gauss-Manin connection. Here, the Brieskorn lattice $\mathcal{H}_{X, 0}^{(0)}=\Omega_{X, 0}^{n+1} / d f \wedge d \Omega_{X, 0}^{n-1}$ plays an important role. The other is the theory of mixed Hodge structure of Steenbrink on the vanishing cohomology $H^{n}\left(X_{\infty}, C\right)$ (cf. (1.2)).

In [7], Scherk and Steenbrink constructed a filtration on $H^{n}\left(X_{\infty}, C\right)$ using $\mathcal{H}_{X}^{(0)}$, and asserted that the filtration coincides with Hodge filtration $\left\{\boldsymbol{F}_{S t}{ }^{\prime}\right\}$ of the mixed Hodge structure. But there is an example (e.g. $\left.f=x^{5}+y^{5}+x^{3} y^{3}\right)$, in which their filtration is not compatible with the monodromy decomposition $H^{n}\left(X_{\infty}, C\right)=\oplus_{\lambda} H^{n}\left(X_{\infty}, C\right)_{\lambda}$, whereas $\left\{F_{s t}^{*}\right\}$ is compatible with it. Here $H^{n}\left(X_{\infty}, C\right)_{\lambda}:=\left\{u \in H^{n}\left(X_{\infty}, C\right):\left(M_{X}-\lambda\right)^{n+1} u\right.$ $=0$ ) and $M_{X}$ is the local monodromy of $f$.

This contradiction comes from the following. In [9], Steenbrink proved that the Hodge subbundle of the flat vector bundle $H_{Y}$ $=\bigsqcup_{t \in S^{*}} H^{n}\left(Y_{t}, C\right)$ can be extended to the origin as a subbundle of Deligne's extension $\mathcal{L}_{Y}^{\prime}$ of $H_{Y}$ (cf. (1.3)). Here, $\bar{f}: Y \rightarrow S$ is a one-parameter projective family. But this limit filtration is not compatible with the monodromy decomposition $H^{n}\left(Y_{\infty}, C\right)=\oplus H^{n}\left(Y_{\infty}, C\right)_{\lambda}$. Following the construction of Schmid, we have to take a base change such that the pullback of $H_{Y}$ has a unipotent monodromy.

In this note, we give a correct formulation of their assertion and an outline of the proof.

I would like to thank Profs. Kyoji Saito and Masaki Kashiwara for helpful discussions.

§ 1. Limit mixed Hodge structure. (1.1) Let $f: C^{n+1}, 0 \rightarrow C, 0$ be a holomorphic function with an isolated singularity. We may assume that $f$ is a polynomial of degree $d$, where we can take $d$ as large as we like, and that $\overline{f^{-1}(0) \subset P^{n+1}}$ is smooth away from the origin. We define $Y:=\left\{\overline{(x, t) \in C^{n+1} \times S: f(x)=t}\right\} \subset P^{n+1} \times S$ and $X:=(B \times S) \cap Y$, where $S:=\{t \in C:|t|<\eta\}$ and $B:=\left\{x \in C^{n+1}:\|x\|<\varepsilon\right\}$. We put $Z:=\boldsymbol{P}^{n+1}$ $\times S, p: Z \rightarrow S$ the natural projection and $\bar{f}:=\left.p\right|_{Y}$. For $1 \gg \varepsilon \gg \eta>0$, $\bar{f}: Y \rightarrow S$ is smooth away from 0 and $f: X \rightarrow S$ is a Milnor fibration, i.e., $f^{\prime}: X^{*}=X-f^{-1}(0) \rightarrow S^{*}=S-\{0\}$ is a $C^{\infty}$-fibration. Hence, $H_{X}$ : $=\left.R^{n} f_{*} C_{X}\right|_{S^{*}}\left(\right.$ resp. $\left.H_{Y}:=\left.R^{n} \bar{f}_{*} C_{Y}\right|_{S^{*}}\right)$ is a local system with a monodromy 
$M_{X}\left(\operatorname{resp} . M_{Y}\right)$.

(1.2) Let $\pi: \tilde{S} \ni \tilde{t}_{\mapsto} \mapsto t=\tilde{t}^{e} \in S$ be an $e$-fold covering of $S$, such that $\tilde{H}_{Y}:=\pi^{*} H_{Y}$ has a unipotent monodromy. We set $X_{\infty}:=X \times{ }_{S} U$ and $Y_{\infty}:=Y \times{ }_{S} U$, where $U \rightarrow S^{*}$ is a universal covering.

(1.3) As was proved by Manin and Deligne (see [3]), the local system $H_{X}$ can be uniquely extended to the origin as a locally free $\mathcal{O}_{S^{-}}$ Module $\mathcal{L}_{X}$ (resp. $\mathcal{L}_{X}^{\prime}$ ) with a regular singular connection $\nabla$, such that $\nabla$ has a simple pole and the residue of $\nabla$ has its eigenvalues in $(-1,0]$ (resp. [0,1)). We have an isomorphism $H^{n}\left(X_{\infty}, C\right) \widetilde{\mathcal{L}_{X}}(0):=\mathcal{L}_{X, 0} / t \mathcal{L}_{X, 0}$ defined by $u \mapsto \exp \left(-\log M_{X} \log t / 2 \pi \sqrt{-1}\right) u$, where we regard $u$ as a multivalued section of $H_{X}$.

We can obtain similar extensions for $H_{Y}, \tilde{H}_{Y}$ and $\tilde{H}_{X}:=\pi^{*} H_{X}$, and denote them by $\mathcal{L}_{Y}, \widetilde{\mathcal{L}}_{Y}$, etc.

(1.4) Proposition (Schmid [8]). Let $\left\{\mathcal{F}^{\circ}\right\}$ be the Hodge subbundles of $H_{Y}$ (i.e., $\left\{\mathcal{F}^{*}(t)\right\}$ is the Hodge filtration of $H^{n}\left(Y_{t}, C\right)$ for $\left.\forall t \in S^{*}\right)$. Then $\widetilde{F}^{\circ}:=\pi^{*} \mathcal{F}^{\circ}$ can be extended to the origin as holomorphic vector subbundles of $\widetilde{\mathcal{L}}_{Y}$, and the limit Hodge filtration $F_{S}{ }_{S} \subset \widetilde{\mathcal{L}}_{Y}(0) \approx H^{n}\left(Y_{\infty}, C\right)$ forms a mixed Hodge structure with the monodromy weight filtration.

(1.5) Proposition (Steenbrink [9]). $H^{n}\left(X_{\infty}, C\right)$ has a mixed Hodge structure such that $i^{*}: H^{n}\left(Y_{\infty}, C\right) \rightarrow H^{n}\left(X_{\infty}, C\right)$ is a morphism of mixed Hodge structures.

§2. Gauss-Manin systems. (2.1) Definition. The Gauss-Manin systems are the integration of systems defined by

$$
\begin{aligned}
& \mathcal{H}_{X}:=\mathscr{H}^{0}\left(\boldsymbol{R} p_{*}^{\prime} D R_{Z^{\prime} / S}\left(\mathscr{B}_{X \mid Z^{\prime}}\right)[n+1]\right), \\
& \mathcal{H}_{Y}:=\mathcal{H}^{0}\left(\boldsymbol{R} p_{*} D R_{Z / S}\left(\mathcal{B}_{Y \mid Z}\right)[n+1]\right),
\end{aligned}
$$

where $Z^{\prime}:=B \times S, p^{\prime}:=p \mid Z^{\prime}, \mathscr{B}_{X \mid Z}:=\mathcal{H}_{[X]}^{1}\left(\mathcal{O}_{Z^{\prime}}\right)$ and $D R_{Z^{\prime} / S}\left(\mathscr{B}_{X \mid Z^{\prime}}\right)$ : $=\Omega_{Z^{\prime} / S} \otimes \mathcal{B}_{X \mid Z^{\prime}}$ (cf. [4]).

$\mathcal{H}_{X}$ (resp. $\mathcal{H}_{Y}$ ) is a holonomic system with a regular singularity such that $D R_{S}\left(\mathcal{H}_{X}\right)=R^{n} f_{*} C_{X}$ (resp. $\left.\mathcal{H}^{0}\left(D R_{S}\left(\mathcal{H}_{Y}\right)\right)=R^{n} \bar{f}_{*} C_{Y}\right)$. We remark that $\mathcal{H}_{X}$ (resp. $\mathcal{H}_{Y}$ ) contains $\mathcal{L}_{X}$ (resp. $\mathcal{L}_{Y}$ ) and the action of $\nabla_{d / d t}$ coincides with the action of $\partial_{t} \in \mathscr{D}_{S}$.

(2.2) Definition.

$$
D R_{Z^{\prime} / S}\left(\mathscr{B}_{X \mid Z^{\prime}}\right)(k):=\left\{\Omega_{Z^{\prime} / S}^{\cdot} \otimes \mathscr{B}_{X \mid Z^{\prime}}(\cdot+k-n-1)\right\}
$$

for $k \in Z_{+}:=\{\mathrm{m} \in \boldsymbol{Z}: m \geq 0\}$, where $\mathscr{B}_{X \mid Z^{\prime}}(m):=\mathscr{D}_{\mathbb{Z}^{\prime}}(m) \delta(f(x)-t)$ and $\mathscr{D}_{Z^{\prime}}(m):=\left\{\sum_{|\nu| \leq m} a_{\nu} \partial^{\nu}\right\} \subset \mathscr{D}_{Z^{\prime}}$ for $m \in Z$. We define $D R_{Z / S}\left(\mathscr{B}_{Y \mid Z}\right)(k)$ and $\mathscr{B}_{Y \mid Z}(m)$ similarly.

(2.3) Definition.

$$
\begin{array}{lll}
\mathcal{H}_{X}^{(k)}:=\operatorname{Im}\left(\mathscr{H}^{0}\left(\boldsymbol{R} p_{*}^{\prime}\left(D R_{Z^{\prime} / S}\left(\mathcal{B}_{X \mid Z^{\prime}}\right)(k)\right)[n+1]\right) \longrightarrow \mathcal{H}_{X}\right) & k \in \boldsymbol{Z}_{+}, \\
\mathcal{H}_{Y}^{(k)}:=\operatorname{Im}\left(\mathcal{H}^{0}\left(\boldsymbol{R} p_{*}\left(D R_{Z / S}\left(\mathscr{B}_{Y \mid Z}\right)(k)\right)[n+1]\right) \longrightarrow \mathcal{H}_{Y}\right) & k \in \boldsymbol{Z}_{+} .
\end{array}
$$

We remark that the natural inclusion $i: X \rightarrow Y$ induces a morphism $i_{\mathscr{D}}^{*}: \mathcal{H}_{Y} \rightarrow \mathcal{H}_{X}$ such that $i_{\mathscr{D}}^{*}\left(\mathcal{H}_{Y}^{(k)}\right) \subset \mathcal{H}_{X}^{(k)}$, and we have $\mathcal{H}_{X}^{(k)}=\partial_{t}^{k} \mathcal{H}_{X}^{(0)}$ for $\forall k \in Z_{+}$. 
(2.4) Proposition (cf. [2]). $\left.\quad \mathcal{H}_{Y}^{(k)}\right|_{S^{*}}$ is a holomorphic subbundle of $H_{Y}$. Furthermore, it coincides with the Hodge bundle $\mathscr{F}^{n-k}$ (cf. (1.4)).

(2.5) Theorem. If the degree $d$ of $f$ is sufficiently large, then we have $i_{\mathscr{D}}^{*}\left(\mathcal{H}_{Y}\right)=\mathcal{H}_{X}, i_{\mathscr{D}}^{*}\left(\mathcal{H}_{Y}^{(k)}\right)=\mathcal{H}_{X}^{(k)}$ for $\forall k \in Z_{+}$and $\mathcal{K}:=\operatorname{Ker} i_{\mathscr{D}}^{*}$ is a free $\mathcal{O}_{S}$-Module of finite rank, i.e.

$$
0 \longrightarrow \mathcal{K}=\oplus \mathcal{O}_{S} \longrightarrow \mathcal{H}_{Y} \longrightarrow \mathcal{H}_{X} \longrightarrow 0
$$

is an exact sequence of $\mathscr{D}_{S}$-Modules.

We remark that $D R_{S}(\mathcal{K}) \subset R^{n} \bar{f}_{*} C_{Y}$ is the sheaf of invariant cycles of $\bar{f}: Y \rightarrow S$ and the exact sequence (2.5.1) does not split if there is an invariant cycle in $H_{X}$.

This theorem follows from the theory of microlocalization (cf. [4]) and the next lemma.

(2.6) Lemma (Scherk [7]). If $d$ is sufficiently large, there is a $C\{t\}$ basis $\left\{w_{i}\right\}_{i=1, \ldots, \mu}$ of $\mathcal{H}_{X, 0}^{(0)}$ such that res $\left(w_{i} /(f-t)\right)$ can be extended to holomorphic relative $n$-forms on $Y-\{0\}$.

Remark. The exact sequence (2.5.1) was found independently by F. Pham.

§3. The Gauss Manin system determines the Hodge filtration.

(3.1) Definition. We define a decreasing filtration $\left\{F_{\mathcal{H}}{ }^{\prime}\right\}$ on $H^{n}\left(X_{\infty}, C\right)$ by

$$
F^{k}{ }_{\mathscr{K}}:=\operatorname{Im}\left(\pi^{*}\left(\mathcal{H}_{X}^{(n-k)} \cap \mathcal{L}_{X}\right) \cap \widetilde{\mathcal{L}}_{X} \longrightarrow \widetilde{\mathcal{L}}_{X}(0) \simeq H^{n}\left(X_{\infty}, C\right)\right),
$$

where $\left.\pi^{*}\left(\mathcal{H}_{X}^{(n-k)}\right) \cap \mathcal{L}_{X}\right)$ is an $\mathcal{O}_{\tilde{S}}$-submodule of $\widetilde{\mathcal{L}}_{X} \otimes \mathcal{O}_{\tilde{S}} \mathcal{O}_{\tilde{s}}\left[\tilde{t}^{-1}\right]$ generated by $\pi^{*} w$ with $w \in \mathcal{H}_{X}^{(n-k)} \cap \mathcal{L}_{X}$.

(3.2) Theorem. We have $\left\{F_{\mathscr{T}}^{*}\right\}=\left\{F_{S t}^{\cdot}\right\}$, where $\left\{F_{S t}^{\cdot}\right\}$ is the Hodge filtration of Steenbrink on $H^{n}\left(X_{\infty}, C\right)$ (cf. (1.5)).

(3.3) Outline of the proof. The inclusion $F_{\mathscr{K}}^{k} \subset F_{S t}^{k}$ is obvious from (1.4), (1.5), (2.4) and (2.5). To prove the reverse inclusion, we need two results : the duality of exponents due to Steenbrink (cf. [5] [9]) and the following lemma due to Kyoji Saito (cf. [11]).

(3.4) Lemma. Let $\left\{w_{i}\right\}$ be a $C\{t\}$ basis of $\mathcal{H}_{X, 0}^{(0)}$ and let $\left\{\gamma_{i}(t)\right\}$ be a basis of multivalued horizontal sections of $\bigsqcup_{t \in S^{*}} H_{n}\left(X_{t}, C\right)$. Then we have $\left(\operatorname{det}\left(\int_{r_{i}(t)} w_{j}\right)\right)^{2}=t^{\mu(n-1)} g(t)$, where $g(t)$ is a holomorphic function such that $g(0) \neq 0$.

The rest of the proof is almost the same as Varchenko [10, Lemma $2]$.

(3.5) Remarks, 1. If we define $\left\{{ }^{\prime} \boldsymbol{F}^{*}\right\}$ by ${ }^{\prime} \boldsymbol{F}^{k}:=\operatorname{Im}\left(\pi^{*} \mathcal{H}_{X}^{(n-k)}\right.$ $\left.\cap \widetilde{\mathcal{L}}_{X} \rightarrow \widetilde{\mathcal{L}}_{X}(0)\right)$, we have ' $F^{k} \supset F_{\mathscr{H}}^{k}$. But the equality does not hold in general (e.g. $f=x^{5}+y^{5}+z^{5}+x^{3} y^{3}$ ).

2. Varchenko defined a similar filtration $\left\{\boldsymbol{F}_{a}^{\cdot}\right\}$ (cf. [10]). But it is different from $\left\{F_{\mathscr{T}}\right\}$, if there exsists $k \leq n-1$ such that $h_{\lambda}^{k, n+1-k} \neq 0$ $(\lambda \neq 1)$ or $h_{1}^{k, n+2-k} \neq 0$ (cf. [6]).

(3.6) Corollary. Hodge number $h_{\lambda}^{p, q}:=\operatorname{dim}_{C} G r_{F}^{p} G r_{p+q}^{W} H^{n}\left(X_{\infty}, C\right)_{\lambda}$ 
and exponents (cf. [5]) are constant under $\mu$-constant deformations (cf. [7]).

\section{References}

[1] Brieskorn, E.: Die Monodromie der isolierten Singularitäten von Hyperflächen. Manuscripta Math., 2, 103-161 (1970).

[2] Brylinski, J. L.: Modules holonomes à singularités régulières et filtration de Hodge, I (preprint).

[3] Deligne, P.: Equations différentielles à points singuliers réguliers. Lect. Notes in Math., vol. 163, Springer (1970).

[4] Pham, F.: Singularités des systèmes différentiels de Gauss-Manin. Progress in Math., 2, Birkhäuser (1979).

[5] Saito, M.: On the exponents and the geometric genus of an isolated hypersurface singularity (to appear in Proceedings of the Symposia in pure Math., 40, A.M.S.).

[6] Scherk, J.: A note on two local Hodge filtrations (to appear).

[7] Scherk, J., and Steenbrink, J.: On the mixed Hodge structure on the cohomology of the Milnor fiber (preprint).

[8] Schmid, W.: Variation of Hodge structure: the singularities of the period mapping. Invent. math., 22, 211-319 (1973).

[9] Steenbrink, J. H. M.: Mixed Hodge structure on the vanishing cohomology. Proceedings of the Nordic Summer School, Oslo, pp. 525-563 (1976).

[10] Varchenko, A.: Asymptotics of holomorphic forms define mixed Hodge structure. Dokl. Akad. Nauk. SSSR, 255 (5), 1035-1038 (1980).

[11] — : Asymptotic mixed Hodge structure on vanishing cohomology. Izv. Akad. Nauk. SSSR, Ser. Math., 45(3), 540-591 (1981). 